\title{
INSERTION OF CONTINUOUS REAL FUNCTIONS ON SPACES, BISPACES, ORDERED SPACES AND POINT-FREE SPACES - A COMMON ROOT
}

\author{
MARIA JOÃO FERREIRA, JAVIER GUTIÉRREZ GARCÍA AND JORGE PICADO
}

\begin{abstract}
We characterize normal and extremally disconnected biframes in terms of the insertion of a continuous real function in between given lower and upper semicontinuous real functions and show this to be the common root of several classical and new insertion results concerning topological spaces, bitopological spaces, ordered topological spaces and locales.
\end{abstract}

KEYWords: Frame, locale, localic real function, insertion theorem, biframe, normal biframe, extremally disconnected biframe, bitopological space, ordered topological space.

AMS Subject Classification (2000): 06D22, 26A15, 54C30, 54D15, 54E55, 54F05.

\section{Introduction}

Various kinds of spaces are characterized in terms of the existence of a continuous real function in between given lower and upper semicontinuous real functions, such as

- normal spaces (Katětov-Tong insertion theorem $([11,25])$ ), and

- extremally disconnected spaces (Stone insertion theorem [26]).

These results rank among the fundamental results in point-set topology and provide as corollaries a separation-type theorem (e.g. in the normal case the celebrated Urysohn Lemma) and an extension-type theorem (e.g. in the normal case the well known Tietze Extension Theorem). The corresponding counterparts in point-free topology were obtained recently (see $[18,9,6]$;

Received January 16, 2009.

The first and third named authors acknowledge financial support from the Centre of Mathematics of the University of Coimbra/FCT. The second named author acknowledges financial support from the Ministry of Education and Science of Spain and FEDER under grant MTM2006-14925-C02-02 and from the University of the Basque Country under grant GIU07/27. 
in [7] and [4] perfectly normal, countably paracompact and completely normal point-free spaces are also characterized via insertion of continuous real functions and [8] presents a nice general point-free setting for arbitrary real functions in which those results can be nicely formalized). There are also in the literature versions of some of these results for bitopological spaces (as the Katětov-Tong version of Priestley for normal bitopological spaces [21] and the separation and the extension-type theorems of Sarma for extremally disconnected bitopological spaces [22]) and ordered topological spaces (Nachbin [17], Priestley [21], Edwards [3]).

The purpose of this paper is to exhibit a natural common root for these results, encompassing at once topological spaces, bitopological spaces, ordered topological spaces, point-free spaces (that is, frames and locales) and pointfree bispaces (that is, biframes) in a single setting. The proper setting for this will be the category of biframes and, specifically, their (relative) lower and upper semicontinuous real functions.

After a brief survey of the required background and the introduction of a few new concepts in biframes, we begin with a new characterization of normal biframes from which it follows a Katětov-Tong-type insertion theorem for biframes $\left(L_{0}, L_{1}, L_{2}\right)$ that generalizes all the above mentioned Katětov-Tongtype insertion results:

- The case $L_{0}=L_{1}=L_{2}$ provides the result for frames [18, 9]. Making further $L_{0}=L_{1}=L_{2}=\mathfrak{T}$ for any topological space $(X, \mathfrak{T})$ provides the classical Katětov-Tong theorem.

- The case $\left(L_{0}, L_{1}, L_{2}\right)=\left(\mathfrak{T}_{1} \vee \mathfrak{T}_{2}, \mathfrak{T}_{1}, \mathfrak{T}_{2}\right)$ for a bitopological space $\left(X, \mathfrak{T}_{1}, \mathfrak{T}_{2}\right)$ provides the Katětov-Tong-type result of Priestley [21] for bitopological spaces.

- The case $\left(L_{0}, L_{1}, L_{2}\right)=(\downarrow \mathfrak{T} \vee \uparrow \mathfrak{T}, \downarrow \mathfrak{T}, \uparrow \mathfrak{T})$ for an ordered topological space $(X, \mathfrak{T}, \leq)$, and some variants of it, provide the Katětov-Tongtype results of Priestley [21] and Nachbin [17] for ordered topological spaces.

Then we do a similar study for the extremally disconnected biframes and Stone insertion theorem. Here the application of our general theorem to bitopological spaces and ordered topological spaces provides new results in the literature. 


\section{Background on frames and biframes}

We shall denote the category of frames and frame homomorphisms by Frm and the corresponding dual category of locales and localic maps by Loc. For general facts concerning frames, locales and various related ideas we refer to [10], [19] and [20]. Here we fix some terminology and notations.

A frame (or locale) $L$ is normal if whenever $a \vee b=1$ there exist $u, v \in L$ satisfying $u \wedge v=0$ and $a \vee u=1=b \vee v$ (equivalently, if there exists $u \in L$ such that $a \vee u=1=b \vee u^{*}$ for the pseudocomplement $u^{*}=\bigvee\{v \mid u \wedge v=0\}$ ). Dually, $L$ is extremally disconnected if whenever $a \wedge b=0$ there exist $u, v \in L$ such that $u \vee v=1$ and $a \wedge u=0=b \wedge v$. Equivalently, if $a^{*} \vee a^{* *}=1$ for every $a \in L$ (which is the same as asserting that the second De Morgan law $(a \wedge b)^{*}=a^{*} \vee b^{*}$ holds in $\left.L\right)$.

A subset $S$ of $L$ is a sublocale of $L$ (i.e. a regular subobject of $L$ in Loc) if, whenever $A \subseteq L, a \in L$ and $s \in S$, then $\bigwedge A \in S$ and $a \rightarrow s \in S$. Each sublocale $S$ of $L$ is a frame itself with $\wedge$ and $\rightarrow$ inherited from $L$ (the top element of $S$ is 1, while the bottom $0_{S}$ of $S$ may differ from 0$)$. It determines the surjection (frame quotient) $c_{S}: L \rightarrow S$ given by $c_{S}(x)=\bigwedge\{s \in S: x \leq$ $s\}$.

The intersection of sublocales is again a sublocale, so that the set of all sublocales is a complete lattice under inclusion. In fact, it is a co-frame, in which $\{1\}$ is the bottom element and $L$ is the top [19].

For notational reasons, we shall make the co-frame of all sublocales into a frame $\mathcal{S}(L)$ by considering the opposite ordering:

$$
S_{1} \leq S_{2} \quad \Leftrightarrow \quad S_{2} \subseteq S_{1}
$$

Thus, given $\left\{S_{i} \in \mathcal{S}(L): i \in I\right\}$, we have

$$
\bigvee_{i \in I} S_{i}=\bigcap_{i \in I} S_{i} \text { and } \bigwedge_{i \in I} S_{i}=\left\{\bigwedge A: A \subseteq \bigcup_{i \in I} S_{i}\right\}
$$

Then $\{1\}$ is the top element and $L$ is the bottom element in $\mathcal{S}(L)$ that we just denote by 1 and 0 , respectively. The pseudocomplement of $S$ in $\mathcal{S}(L)$ will standardly be denoted by $S^{*}$.

Among the important examples of sublocales are the closed and open sublocales

$$
\mathfrak{c}_{L}(a)=\uparrow a=\{b \in L: a \leq b\} \quad \text { and } \quad \mathfrak{o}_{L}(a)=\{a \rightarrow b: b \in L\}
$$


for every $a \in L$. We denote them just by $\mathfrak{c}(a)$ and $\mathfrak{o}(a)$ whenever there is no more than one frame $L$ in question. The quotients $c_{\mathfrak{c}(a)}$ and $c_{\mathfrak{o}(a)}$ are given by

$$
c_{\mathfrak{c}(a)}(x)=a \vee x \quad \text { and } \quad c_{\mathfrak{o}(a)}(x)=a \rightarrow x .
$$

We shall freely use the following properties:

Properties 1.1. For every $a \in L$ we have:

(1) The map $a \mapsto \mathfrak{c}(a)$ is a frame embedding $\mathfrak{c}_{L}: L \rightarrow \mathcal{S}(L)$.

(2) $\mathfrak{c}(a) \vee \mathfrak{o}(a)=1$ and $\mathfrak{c}(a) \wedge \mathfrak{o}(a)=0$. In particular:

- $\mathfrak{o}(a) \geq \mathfrak{c}(b)$ if and only if $a \wedge b=0$,

- $\mathfrak{o}(a) \leq \mathfrak{c}(b)$ if and only if $a \vee b=1$,

and, for any $S \in \mathcal{S}(L), S \vee \mathfrak{c}(a)=1$ if and only if $S \geq \mathfrak{o}(a)$.

For any frame $L$, we denote by $\mathfrak{c} L$ the subframe of $\mathcal{S}(L)$ consisting of all closed sublocales of $L$ (by Properties 1.1 (1), $L$ and $\mathfrak{c} L$ are isomorphic) and we denote by $\mathfrak{o} L$ the subframe of $\mathcal{S}(L)$ generated by all open sublocales of $L$.

Recall tat a subset $M$ of a frame $L$ is a subframe of $L$ if it is closed under finite meets and arbitrary joins (in particular, $0,1 \in M$ ). A biframe [2] is a triple $\left(L_{0}, L_{1}, L_{2}\right)$ in which $L_{0}$ is a frame and $L_{1}$ and $L_{2}$ are subframes of $L_{0}$, which together generate it. This means that any element $x$ of $L_{0}$ can be expressed as a join of finite meets of elements of $L_{1} \cup L_{2}$, that is, $x=\bigvee_{i \in I}\left(a_{i} \wedge b_{i}\right)$ for some $a_{i} \in L_{1}$ and $b_{i} \in L_{2}$.

A biframe homomorphism [2] $f:\left(L_{0}, L_{1}, L_{2}\right) \rightarrow\left(M_{0}, M_{1}, M_{2}\right)$ is a frame homomorphism $f: L_{0} \rightarrow M_{0}$ for which $f\left(L_{i}\right) \subseteq M_{i}(i=1,2)$. Biframes and biframe homomorphisms form the category BiFrm of biframes [2]. For more information on BiFrm we refer to [23].

Given a biframe $\left(L_{0}, L_{1}, L_{2}\right)$, we shall denote by $\left(\mathfrak{c} L_{0}\right)_{i}(i=1,2)$ the system $\left\{\mathfrak{c}_{L_{0}}(a) \mid a \in L_{i}\right\}$ of all closed sublocales of $L_{0}$ determined by elements $a \in L_{i}$. Similarly, $\left(\mathfrak{o} L_{0}\right)_{i}(i=1,2)$ denotes the set $\left\{\mathfrak{o}_{L_{0}}(a) \mid a \in L_{i}\right\}$. Note that $\left(\mathfrak{c} L_{0},\left(\mathfrak{c} L_{0}\right)_{1},\left(\mathfrak{c} L_{0}\right)_{2}\right)$ is a biframe isomorphic to $\left(L_{0}, L_{1}, L_{2}\right)$.

The biframe

$$
\left(\mathcal{S}_{0}\left(L_{0}\right), \mathcal{S}_{1}\left(L_{1}\right), \mathcal{S}_{2}\left(L_{2}\right)\right)
$$

is defined as follows (cf. Definition 1.12 in [23], written in terms of congruences):

$\mathcal{S}_{i}\left(L_{i}\right)(i=1,2)$ is the subframe of $\mathcal{S}\left(L_{0}\right)$ generated by $\left(\mathfrak{c} L_{0}\right)_{i} \cup\left(\mathfrak{o} L_{0}\right)_{j}(j=$ $1,2, j \neq i)$ and $\mathcal{S}_{0}\left(L_{0}\right)$ is the subframe of $\mathcal{S}\left(L_{0}\right)$ generated by $\mathcal{S}_{1}\left(L_{1}\right) \cup \mathcal{S}_{2}\left(L_{2}\right)$. 
The map $\left(L_{0}, L_{1}, L_{2}\right) \rightarrow\left(\mathcal{S}_{0}\left(L_{0}\right), \mathcal{S}_{1}\left(L_{1}\right), \mathcal{S}_{2}\left(L_{2}\right)\right)$ given by $a \mapsto \mathfrak{c}_{L_{0}}(a)$ for all $a \in L_{0}$ is a biframe embedding.

Another important example of a biframe is the biframe of reals

$$
\left(\mathfrak{L}(\mathbb{R}), \mathfrak{L}_{l}(\mathbb{R}), \mathfrak{L}_{u}(\mathbb{R})\right)
$$

where $\mathfrak{L}(\mathbb{R})$ is the frame of reals $([1],[15])$ defined by generators $(p,-)$ and $(-, q)(p, q \in \mathbb{Q})$ and relations

(R1) $(p,-) \wedge(-, q)=0$ whenever $p \geq q$,

(R2) $(p,-) \vee(-, q)=1$ whenever $p<q$,

(R3) $(p,-)=\bigvee_{r>p}(r,-)$,

(R4) $(-, q)=\bigvee_{s<q}(-, s)$,

(R5) $\bigvee_{p \in \mathbb{Q}}(p,-)=1$,

(R6) $\bigvee_{q \in \mathbb{Q}}(-, q)=1$,

and

$$
\begin{aligned}
\mathfrak{L}_{u}(\mathbb{R}) & =\langle\{(p,-): p \in \mathbb{Q},(p,-) \text { satisfy }(\mathrm{R} 3) \text { and }(\mathrm{R} 5) \text { for all } p \in \mathbb{Q}\}\rangle, \\
\mathfrak{L}_{l}(\mathbb{R}) & =\langle\{(-, q): q \in \mathbb{Q},(-, q) \text { satisfy }(\mathrm{R} 4) \text { and }(\mathrm{R} 6) \text { for all } q \in \mathbb{Q}\}\rangle .
\end{aligned}
$$

A biframe $\left(L_{0}, L_{1}, L_{2}\right)$ is called normal $[23,24]$ if, whenever $a \vee b=1$ for some $a \in L_{1}$ and $b \in L_{2}$, there exist $u \in L_{2}$ and $v \in L_{1}$ such that $u \wedge v=0$ and $a \vee u=1=b \vee v$.

For $a \in L_{i}(i=1,2)$ let $a^{\bullet}$ be the largest $b \in L_{j}(j=1,2, j \neq i)$ for which $a \wedge b=0$. Normality can then be equivalently expressed by the condition: whenever $a \vee b=1$ for $a \in L_{i}$ and $b \in L_{j}$, there exists $u \in L_{j}$ such that $a \vee u=1=b \vee u^{\bullet}$.

We define sub-bilocales $\left(S_{0}, S_{1}, S_{2}\right)$ of a biframe $\left(L_{0}, L_{1}, L_{2}\right)$ as its subobjects in the dual category of BiFrm. Thus each $\left(S_{0}, S_{1}, S_{2}\right)$ is given by a regular epimorphism $p_{\left(S_{0}, S_{1}, S_{2}\right)}:\left(L_{0}, L_{1}, L_{2}\right) \rightarrow\left(S_{0}, S_{1}, S_{2}\right)$ in BiFrm, that is, an onto frame homomorphism $p_{\left(S_{0}, S_{1}, S_{2}\right)}: L_{0} \rightarrow S_{0}$ such that $p_{\left(S_{0}, S_{1}, S_{2}\right)}\left(L_{i}\right) \subseteq S_{i}(i=$ $1,2)$.

Remark 1.2. Given a subframe $M$ and a sublocale $S$ of $L$, the set $c_{S}(M)$ is a subframe of $S$. In particular, for any $x \in L$, the sets $x \vee M=\{x \vee m \mid$ $m \in M\}=c_{\mathfrak{c}_{L}(x)}(M)$ and $x \rightarrow M=\{x \rightarrow m \mid m \in M\}=c_{\mathfrak{o}_{L}(x)}(M)$ are subframes of $\mathfrak{c}_{L}(x)$ and $\mathfrak{o}_{L}(x)$, respectively. Note that if $x \in M$ then $x \vee M$ is just $\mathfrak{c}_{M}(x)$.

Therefore, for each $x \in L_{0}, \mathfrak{c}^{b}(x)=\left(\mathfrak{c}_{L_{0}}(x), x \vee L_{1}, x \vee L_{2}\right)$ is a sub-bilocale of $\left(L_{0}, L_{1}, L_{2}\right)$ with $p_{\mathfrak{c}^{b}(x)}: a \mapsto x \vee a$. We refer to these as the closed sub-bilocales 
of $\left(L_{0}, L_{1}, L_{2}\right)$. In particular for any $x \in L_{1}, \mathfrak{c}^{b}(x)=\left(\mathfrak{c}_{L_{0}}(x), \mathfrak{c}_{L_{1}}(x), x \vee L_{2}\right)$ and for any $x \in L_{2}, \mathfrak{c}^{b}(x)=\left(\mathfrak{c}_{L_{0}}(x), x \vee L_{1}, \mathfrak{c}_{L_{2}}(x)\right)$.

Further, for each $x \in L_{0}, \mathfrak{o}^{b}(x)=\left(\mathfrak{o}_{L_{0}}(x), x \rightarrow L_{1}, x \rightarrow L_{2}\right)$ is a subbilocale of $\left(L_{0}, L_{1}, L_{2}\right)$ with $p_{\mathfrak{o}^{b}(x)}: y \mapsto(x \rightarrow y)$. We refer to these as the open sub-bilocales of $\left(L_{0}, L_{1}, L_{2}\right)$.

\section{Real functions on frames and biframes}

In general topology one sometimes deals with arbitrary (not necessarily continuous) real-valued functions on a topological space $X$. This is also possible in the point-free setting with the approach recently introduced in [8] (which extends the approach to point-free continuous real functions of Banaschewski [1]):

Let $L$ be a frame. A real function on $L$ is an $f \in \mathrm{F}(L)=\operatorname{Frm}(\mathfrak{L}(\mathbb{R}), \mathcal{S}(L))$. It is

(1) lower semicontinuous if $f\left(\mathfrak{L}_{u}(\mathbb{R})\right) \subseteq \mathfrak{c} L$,

(2) upper semicontinuous if $f\left(\mathfrak{L}_{l}(\mathbb{R})\right) \subseteq \mathfrak{c} L$,

(3) continuous if $f(\mathfrak{L}(\mathbb{R})) \subseteq \mathfrak{c} L$.

$\mathrm{F}(L)$ is partially ordered by

$$
\begin{array}{rlll}
f \leq g & \Leftrightarrow \quad f(p,-) \leq g(p,-) & \text { for every } p \in \mathbb{Q} \\
& \Leftrightarrow \quad g(-, q) \leq f(-, q) & \text { for every } q \in \mathbb{Q} .
\end{array}
$$

Now, given a biframe $\left(L_{0}, L_{1}, L_{2}\right)$, a real function on $\left(L_{0}, L_{1}, L_{2}\right)$ is a frame homomorphism $f: \mathfrak{L}(\mathbb{R}) \rightarrow \mathcal{S}_{0}\left(L_{0}\right)$ such that

$$
f:\left(\mathfrak{L}(\mathbb{R}), \mathfrak{L}_{l}(\mathbb{R}), \mathfrak{L}_{u}(\mathbb{R})\right) \rightarrow\left(\mathcal{S}_{0}\left(L_{0}\right), \mathcal{S}_{1}\left(L_{1}\right), \mathcal{S}_{2}\left(L_{2}\right)\right)
$$

is a biframe homomorphism. Further:

(1) $f$ is $L_{1}$-usc if $f\left(\mathfrak{L}_{l}(\mathbb{R})\right) \subseteq\left(\mathfrak{c} L_{0}\right)_{1}$,

(2) $f$ is $L_{2}$-lsc if $f\left(\mathfrak{L}_{u}(\mathbb{R})\right) \subseteq\left(\mathfrak{c} L_{0}\right)_{2}$.

The following is obvious since $f(p, q)=f(p,-) \wedge f(-, q)$ :

Proposition 2.1. Let $f$ be a real function on a biframe $\left(L_{0}, L_{1}, L_{2}\right)$. Then $f$ is $L_{1}$-usc and $L_{2}$-lsc if and only if $f$ is continuous in $L_{0}$ and

$$
\mathfrak{c}_{L_{0}}{ }^{-1} \circ f:\left(\mathfrak{L}(\mathbb{R}), \mathfrak{L}_{l}(\mathbb{R}), \mathfrak{L}_{u}(\mathbb{R})\right) \rightarrow\left(L_{0}, L_{1}, L_{2}\right)
$$

is a biframe homomorphism. 
Note that, in particular, " $L_{1}$-usc $+L_{2}$-lsc" is stronger than "continuous in $L_{0} "$

Given a complemented $S \in \mathcal{S}_{i}\left(L_{i}\right)(i=1,2)$ (i.e. such that $S^{\bullet} \vee S=1$ ), the characteristic map $\chi_{S}: \mathfrak{L}(\mathbb{R}) \rightarrow \mathcal{S}_{0}\left(L_{0}\right)$ is defined by

$$
\chi_{S}(p,-)=\left\{\begin{array}{ll}
1 & \text { if } p<0, \\
S^{\bullet} & \text { if } 0 \leq p<1, \\
0 & \text { if } p \geq 1,
\end{array} \quad \text { and } \quad \chi_{S}(-, q)= \begin{cases}0 & \text { if } q \leq 0 \\
S & \text { if } 0<q \leq 1 \\
1 & \text { if } q>1\end{cases}\right.
$$

for every $p, q \in \mathbb{Q}$. Then,

(1) $\chi_{S}$ is $L_{1}$-usc if and only if $S \in\left(\mathfrak{c} L_{0}\right)_{1}$,

(2) $\chi_{S}$ is $L_{2}$-lsc if and only if $S \in\left(\mathfrak{o} L_{0}\right)_{2}$.

\section{Scales}

A way of generating continuous real functions on frames by the so-called scales has been described in detail in [1] (cf. also [8]). In what follows we show how they can be adapted to real functions on biframes. We shall write $\mathfrak{c}_{0}, \mathfrak{c}_{0}(a)$ and $\mathfrak{o}_{0}(a)$ instead of $\mathfrak{c}_{L_{0}}, \mathfrak{c}_{L_{0}}(a)$ and $\mathfrak{o}_{L_{0}}(a)$, respectively.

Let $\left(L_{0}, L_{1}, L_{2}\right)$ be a biframe. We say that a family $\mathcal{A}=\left\{a_{p} \mid p \in \mathbb{Q}\right\} \subseteq L_{0}$ is ascending (resp. descending) if $p<q$ implies $a_{p} \leq a_{q}$ (resp. $a_{p} \geq a_{q}$ ).

An ascending family $\mathcal{A}=\left\{a_{p} \mid p \in \mathbb{Q}\right\} \subseteq L_{1}$ is an

- upper $L_{1}$-scale if $\bigvee_{p \in \mathbb{Q}} a_{p}=1$ and $\bigvee_{p \in \mathbb{Q}} \mathfrak{o}_{0}\left(a_{p}\right)=1$,

- $L_{1}$-scale if $\bigvee_{p \in \mathbb{Q}} a_{p}=1=\bigvee_{p \in \mathbb{Q}} a_{p}^{\bullet}$ and $a_{p}^{\bullet} \vee a_{q}=1$ whenever $p<q$,

and, dually, a descending family $\mathcal{B}=\left\{b_{p} \mid p \in \mathbb{Q}\right\} \subseteq L_{2}$ is an

- lower $L_{2}$-scale if $\bigvee_{p \in \mathbb{Q}} b_{p}=1$ and $\bigvee_{p \in \mathbb{Q}} \mathfrak{o}_{0}\left(b_{p}\right)=1$,

- $L_{2}$-scale if $\bigvee_{p \in \mathbb{Q}} b_{p}=1=\bigvee_{p \in \mathbb{Q}} b_{p}^{\bullet}$ and $b_{p} \vee b_{q}^{\bullet}=1$ whenever $p<q$.

Lemma 3.1. (1) Let $\mathcal{B}=\left\{b_{p} \mid p \in \mathbb{Q}\right\}$ be a lower $L_{2}$-scale. The formulas

$$
f(p,-)=\bigvee_{r>p} \mathfrak{c}_{0}\left(b_{r}\right) \quad \text { and } \quad f(-, q)=\bigvee_{s<q} \mathfrak{o}_{0}\left(b_{s}\right)
$$

determine an $L_{2}$-lsc real function on $\left(L_{0}, L_{1}, L_{2}\right)$.

(2) Let $\mathcal{A}=\left\{a_{p} \mid p \in \mathbb{Q}\right\}$ be an upper $L_{1}$-scale. The formulas

$$
g(p,-)=\bigvee_{r>p} \mathfrak{o}_{0}\left(a_{r}\right) \quad \text { and } \quad g(-, q)=\bigvee_{s<q} \mathfrak{c}_{0}\left(a_{s}\right)
$$

determine an $L_{1}$-usc real function on $\left(L_{0}, L_{1}, L_{2}\right)$. 
Proof: (1) It suffices to show that $f$ is a frame homomorphism $\mathfrak{L}(\mathbb{R}) \rightarrow$ $\mathcal{S}_{0}\left(L_{0}\right)$, since the rest is obvious by the definition of $f$. For that, we have to check that the map $f$ turns the defining relations $(\mathrm{R} 1)-(\mathrm{R} 6)$ of $\mathfrak{L}(\mathbb{R})$ into identities in $\mathcal{S}_{0}\left(L_{0}\right)$ :

(R1) If $p \geq q$ then $f(p,-) \wedge f(-, q) \leq \mathfrak{c}_{0}\left(b_{p}\right) \wedge \mathfrak{o}_{0}\left(b_{q}\right) \leq \mathfrak{c}_{0}\left(b_{q}\right) \wedge \mathfrak{o}_{0}\left(b_{q}\right)=0$.

$(\mathrm{R} 2)$ If $p<r<s<q$ then $f(p,-) \vee f(-, q) \geq \mathfrak{c}_{0}\left(b_{r}\right) \vee \mathfrak{o}_{0}\left(b_{s}\right) \geq \mathfrak{c}_{0}\left(b_{s}\right) \vee \mathfrak{o}_{0}\left(b_{s}\right)=$ 1 .

(R3) $\bigvee_{r>p} f(r,-)=\bigvee_{r>p} \bigvee_{s>r} \mathfrak{c}_{0}\left(b_{s}\right)=\bigvee_{s>p} \mathfrak{c}_{0}\left(b_{s}\right)=f(p,-)$

(R4) $\bigvee_{s<q} f(-, s)=\bigvee_{s<q} \bigvee_{r<s} \mathfrak{o}_{0}\left(b_{r}\right)=\bigvee_{r<q} \mathfrak{o}_{0}\left(b_{r}\right)=f(-, q)$.

(R5) $\bigvee_{p \in \mathbb{Q}} f(p,-)=\bigvee_{p \in \mathbb{Q}} \bigvee_{r>p} \mathfrak{c}_{0}\left(b_{r}\right)=\bigvee_{r \in \mathbb{Q}} \mathfrak{c}_{0}\left(b_{r}\right)=\mathfrak{c}_{0}\left(\bigvee_{r \in \mathbb{Q}} b_{r}\right)=\mathfrak{c}_{0}(1)=$ 1 .

(R6) $\bigvee_{q \in \mathbb{Q}} f(-, q)=\bigvee_{q \in \mathbb{Q}} \bigvee_{s<q} \mathfrak{o}\left(b_{s}\right)=\bigvee_{q \in \mathbb{Q}} \mathfrak{o}\left(b_{q}\right)=1$

(2) Similar to (1).

Lemma 3.2. Let $\mathcal{B}=\left\{b_{p} \mid p \in \mathbb{Q}\right\}$ be an $L_{2}$-scale. The formulas

$$
h(p,-)=\bigvee_{r>p} \mathfrak{c}_{0}\left(b_{r}\right) \quad \text { and } \quad h(-, q)=\bigvee_{s<q} \mathfrak{c}_{0}\left(b_{s}^{\bullet}\right)
$$

determine an $L_{1}$-usc and $L_{2}$-lsc real function on $\left(L_{0}, L_{1}, L_{2}\right)$.

Proof: It suffices to show that $h$ is a frame homomorphism $\mathfrak{L}(\mathbb{R}) \rightarrow \mathcal{S}_{0}\left(L_{0}\right)$, since the rest is obvious by the definition of $h$. As before, it has to be checked that $h$ turns the defining relations $(\mathrm{R} 1)-(\mathrm{R} 6)$ of $\mathfrak{L}(\mathbb{R})$ into identities in $\mathcal{S}_{0}\left(L_{0}\right)$ :

(R1) If $p \geq q$ then $h(p,-) \wedge h(-, q) \leq \mathfrak{c}_{0}\left(b_{p}\right) \wedge \mathfrak{c}_{0}\left(b_{q}^{\bullet}\right) \leq \mathfrak{c}_{0}\left(b_{q}\right) \wedge \mathfrak{c}_{0}\left(b_{q}^{\bullet}\right)=0$.

(R2) If $p<r<s<q$ then $h(p,-) \vee h(-, q) \geq \mathfrak{c}_{0}\left(b_{r}\right) \vee \mathfrak{c}_{0}\left(b_{s}^{\bullet}\right)=\mathfrak{c}_{0}\left(b_{r} \vee b_{s}^{\bullet}\right)=$ $\mathfrak{c}_{0}(1)=1$.

(R3) $\bigvee_{r>p} h(r,-)=\bigvee_{r>p} \bigvee_{s>r} \mathfrak{c}_{0}\left(b_{s}\right)=\bigvee_{s>p} \mathfrak{c}_{0}\left(b_{s}\right)=h(p,-)$.

(R5) $\bigvee_{p \in \mathbb{Q}} h(p,-)=\bigvee_{p \in \mathbb{Q}} \bigvee_{r>p} \mathfrak{c}_{0}\left(b_{r}\right)=\bigvee_{r \in \mathbb{Q}} \mathfrak{c}_{0}\left(b_{r}\right)=\mathfrak{c}_{0}\left(\bigvee_{r \in \mathbb{Q}} b_{r}\right)=\mathfrak{c}_{0}(1)=$ 1.

(R4) and (R6) may be proved analogously to (R3) and (R5), respectively.

\section{Katětov-Tong type insertion}

Lemma 4.1. A biframe $\left(L_{0}, L_{1}, L_{2}\right)$ is normal if and only if for any countable sets $\left\{a_{k}\right\}_{k \in \mathbb{N}} \subseteq L_{1}$ and $\left\{b_{k}\right\}_{k \in \mathbb{N}} \subseteq L_{2}$ satisfying $\bigwedge_{k \in \mathbb{N}} a_{k} \in L_{1}, \bigwedge_{k \in \mathbb{N}} b_{k} \in L_{2}$, $a_{k} \vee\left(\bigwedge_{\ell \in \mathbb{N}} b_{\ell}\right)=1=b_{k} \vee\left(\bigwedge_{\ell \in \mathbb{N}} a_{\ell}\right)$ for all $k \in \mathbb{N}$, there exists $u \in L_{2}$ such that $a_{k} \vee u=1=b_{k} \vee u^{\bullet}$ for every $k \in \mathbb{N}$. 
Proof: Let $\left(L_{0}, L_{1}, L_{2}\right)$ be a normal biframe. Then, for each $k \in \mathbb{N}, a_{k} \vee$ $\left(\bigwedge_{\ell \in \mathbb{N}} b_{\ell}\right)=1$ implies, by normality, the existence of $v_{k} \in L_{2}$ satisfying $a_{k} \vee v_{k}=1$ and $\left(\bigwedge_{\ell \in \mathbb{N}} b_{\ell}\right) \vee v_{k}^{\bullet}=1$. Similarly, $b_{k} \vee\left(\bigwedge_{\ell \in \mathbb{N}} a_{\ell}\right)=1$ implies the existence of $w_{k} \in L_{1}$ such that $b_{k} \vee w_{k}=1$ and $\left(\bigwedge_{\ell \in \mathbb{N}} a_{\ell}\right) \vee w_{k}^{\bullet}=1$. Then, for each $k \in \mathbb{N}$, we have

$$
a_{k} \vee\left(\bigwedge_{m=1}^{k} w_{m}^{\bullet}\right) \geq\left(\bigwedge_{\ell \in \mathbb{N}} a_{\ell}\right) \vee\left(\bigwedge_{m=1}^{k} w_{m}^{\bullet}\right)=\bigwedge_{m=1}^{k}\left(\left(\bigwedge_{\ell \in \mathbb{N}} a_{\ell}\right) \vee w_{m}^{\bullet}\right)=1
$$

and, similarly,

$$
b_{k} \vee\left(\bigwedge_{m=1}^{k} v_{m}^{\bullet}\right) \geq\left(\bigwedge_{\ell \in \mathbb{N}} b_{\ell}\right) \vee\left(\bigwedge_{m=1}^{k} v_{m}^{\bullet}\right)=\bigwedge_{m=1}^{k}\left(\left(\bigwedge_{\ell \in \mathbb{N}} b_{\ell}\right) \vee v_{m}^{\bullet}\right)=1 .
$$

Now define, for each $k \in \mathbb{N}, u_{k}:=v_{k} \wedge \bigwedge_{m=1}^{k} w_{m}^{\bullet} \in L_{2}$ and $z_{k}:=w_{k} \wedge$ $\bigwedge_{m=1}^{k} v_{m}^{\bullet} \in L_{1}$ and consider $u:=\bigvee_{k \in \mathbb{N}} u_{k} \in L_{2}$ and $z:=\bigvee_{k \in \mathbb{N}} z_{k} \in L_{1}$. Evidently,

$$
a_{k} \vee u \geq a_{k} \vee u_{k}=\left(a_{k} \vee v_{k}\right) \wedge\left(a_{k} \vee\left(\bigwedge_{m=1}^{k} w_{m}^{\bullet}\right)\right)=1
$$

and

$$
b_{k} \vee z \geq b_{k} \vee z_{k}=\left(b_{k} \vee w_{k}\right) \wedge\left(b_{k} \vee\left(\bigwedge_{m=1}^{k} v_{m}^{\bullet}\right)\right)=1
$$

Furthermore,

$$
u \wedge z=\bigvee_{k \in \mathbb{N}} \bigvee_{\ell \in \mathbb{N}}\left(u_{k} \wedge z_{\ell}\right)=\bigvee_{k \in \mathbb{N}} \bigvee_{\ell \in \mathbb{N}}\left(v_{k} \wedge w_{\ell} \wedge \bigwedge_{m=1}^{k} w_{m}^{\bullet} \wedge \bigwedge_{m=1}^{l} v_{m}^{\bullet}\right)=0
$$

Thus $z \leq u^{\bullet}$ and, consequently, $b_{k} \vee u^{\bullet} \geq b_{k} \vee z=1$.

The converse is easy: for each $a \in L_{1}$ and $b \in L_{2}$ with $a \vee b=1$, it suffices to apply the hypothesis to the one-element families $\{a\}$ and $\{b\}$.

Let $\left\{\alpha_{k} \mid k \in \mathbb{N}\right\}$ be an enumeration of $\mathbb{Q}$. Then:

Lemma 4.2. Let $f$ and $g$ be real functions on a normal biframe $\left(L_{0}, L_{1}, L_{2}\right)$. If $g$ is $L_{1}$-usc, $f$ is $L_{2}$-lsc and $g \leq f$ then there exists $\left\{u_{\alpha_{k}}\right\}_{k \in \mathbb{N}} \subseteq L_{2}$ such that

$$
\begin{aligned}
\left(q>\alpha_{k}\right) & \Rightarrow\left(g(-, q) \vee \mathfrak{c}_{0}\left(u_{\alpha_{k}}\right)=1\right), \\
\left(p<\alpha_{k}\right) & \Rightarrow\left(f(p,-) \vee \mathfrak{c}_{0}\left(u_{\alpha_{k}}^{\bullet}\right)=1\right), \\
\left(\alpha_{k_{1}}<\alpha_{k_{2}}\right) & \Rightarrow\left(u_{\alpha_{k_{1}}} \vee u_{\alpha_{k_{2}}}^{\bullet}=1\right) .
\end{aligned}
$$


Proof: We prove this by showing, using induction over $\mathbb{N}$, that, for every $i \in \mathbb{N}$, there exists $\left\{u_{\alpha_{k}}\right\}_{k \leq i} \subseteq L_{2}$ such that

$$
\begin{aligned}
\left(q>\alpha_{k}\right) & \Rightarrow\left(g(-, q) \vee \mathfrak{c}_{0}\left(u_{\alpha_{k}}\right)=1\right), \\
\left(p<\alpha_{k}\right) & \Rightarrow\left(f(p,-) \vee \mathfrak{c}_{0}\left(u_{\alpha_{k}}^{\bullet}\right)=1\right), \\
\left(\alpha_{k_{1}}<\alpha_{k_{2}}\right) & \Rightarrow\left(u_{\alpha_{k_{1}}} \vee u_{\alpha_{k_{2}}}^{\bullet}=1\right), \text { for all } k_{1}, k_{2} \leq i .
\end{aligned}
$$

Since $g \leq f$, we have

$$
\begin{aligned}
\mathfrak{c}_{0}{ }^{-1}(g(-, q)) \vee\left(\bigwedge_{p<\alpha_{1}} \mathfrak{c}_{0}{ }^{-1}(f(p,-))\right) & \geq \mathfrak{c}_{0}^{-1}(g(-, q)) \vee \mathfrak{c}_{0}{ }^{-1}\left(f\left(\alpha_{1},-\right)\right) \\
& =\mathfrak{c}_{0}{ }^{-1}\left(g(-, q) \vee f\left(\alpha_{1},-\right)\right) \\
& \geq \mathfrak{c}_{0}{ }^{-1}\left(g(-, q) \vee g\left(\alpha_{1},-\right)\right)=1
\end{aligned}
$$

for every $q>\alpha_{1}$. Similarly, $\left(\bigwedge_{q>\alpha_{1}} \mathfrak{c}_{0}{ }^{-1}(g(-, q))\right) \vee \mathfrak{c}_{0}{ }^{-1}(f(p,-))=1$ for every $p<\alpha_{1}$. Then, by Lemma 4.1, there exists $u_{\alpha_{1}} \in L_{2}$ satisfying $\mathfrak{c}_{0}{ }^{-1}(g(-, q)) \vee$ $u_{\alpha_{1}}=1$, for every $q>\alpha_{1}$, and $\mathfrak{c}_{0}{ }^{-1}(f(p,-)) \vee u_{\alpha_{1}}^{\bullet}=1$, for every $p<\alpha_{1}$, which shows the first step of the induction.

Now, consider $i \in \mathbb{N}$, and assume, by inductive hypothesis, that for any $k<i$ there is $u_{\alpha_{k}} \in L_{2}$ satisfying $g(-, q) \vee \mathfrak{c}_{0}\left(u_{\alpha_{k}}\right)=1$, for every $q>\alpha_{k}$, $f(p,-) \vee \mathfrak{c}_{0}\left(u_{\alpha_{k}}^{\bullet}\right)=1$, for every $p<\alpha_{k}$, and

$$
\left(\alpha_{k_{1}}<\alpha_{k_{2}}\right) \Rightarrow\left(u_{\alpha_{k_{1}}} \vee u_{\alpha_{k_{2}}}^{\bullet}=1\right) \text {, for all } k_{1}, k_{2} \leq i-1 \text {. }
$$

Further, define

$$
\begin{aligned}
& \left\{a_{\ell}\right\}_{\ell \in \mathbb{N}}:=\left\{\mathfrak{c}_{0}^{-1}(g(-, q)) \mid q>\alpha_{i}\right\} \cup\left\{u_{\alpha_{k}}^{\bullet} \mid k<i, \alpha_{i}<\alpha_{k}\right\}, \\
& \left\{b_{\ell}\right\}_{\ell \in \mathbb{N}}:=\left\{\mathfrak{c}_{0}{ }^{-1}(f(p,-)) \mid p<\alpha_{i}\right\} \cup\left\{u_{\alpha_{k}} \mid k<i, \alpha_{k}<\alpha_{i}\right\} ;
\end{aligned}
$$

Then $\left\{a_{\ell}\right\}_{\ell \in \mathbb{N}}$ and $\left\{b_{\ell}\right\}_{\ell \in \mathbb{N}}$ satisfy the conditions of Lemma 4.1:

(1) For each $q>\alpha_{i}$,

$$
\mathfrak{c}_{0}{ }^{-1}(g(-, q)) \vee\left(\bigwedge_{p<\alpha_{i}} \mathfrak{c}_{0}^{-1}(f(p,-)) \wedge \bigwedge_{k<i, \alpha_{k}<\alpha_{i}} u_{\alpha_{k}}\right)=1
$$

since $\mathfrak{c}_{0}^{-1}(g(-, q)) \vee\left(\bigwedge_{p<\alpha_{i}} \mathfrak{c}_{0}^{-1}(f(p,-))\right)=1$ (again because $g \leq f$ ) and, by inductive hypothesis,

$$
\mathfrak{c}_{0}{ }^{-1}(g(-, q)) \vee\left(\bigwedge_{k<i, \alpha_{k}<\alpha_{i}} u_{\alpha_{k}}\right)=\bigwedge_{k<i, \alpha_{k}<\alpha_{i}}\left(\mathfrak{c}_{0}^{-1}(g(-, q)) \vee u_{\alpha_{k}}\right)=1 .
$$

(2) For each $k<i$ such that $\alpha_{i}<\alpha_{k}$,

$$
u_{\alpha_{k}}^{\bullet} \vee\left(\bigwedge_{p<\alpha_{i}} \mathfrak{c}_{0}{ }^{-1}(f(p,-)) \wedge \bigwedge_{m<i, \alpha_{m}<\alpha_{i}} u_{\alpha_{m}}\right)=1
$$


since $u_{\alpha_{k}}^{\bullet} \vee\left(\bigwedge_{p<\alpha_{i}} \mathfrak{c}_{0}{ }^{-1}(f(p,-))\right) \geq u_{\alpha_{k}}^{\bullet} \vee \mathfrak{c}_{0}{ }^{-1}\left(f\left(\alpha_{i},-\right)\right)=1$ and, by inductive hypothesis,

$$
u_{\alpha_{k}}^{\bullet} \vee\left(\bigwedge_{m<i, \alpha_{m}<\alpha_{i}} u_{\alpha_{m}}\right)=\bigwedge_{m<i, \alpha_{m}<\alpha_{i}}\left(u_{\alpha_{k}}^{\bullet} \vee u_{\alpha_{m}}\right)=1
$$

(3) Similarly to (1) and (2), respectively, one can prove that, for each $p<\alpha_{i}$,

$$
\left(\bigwedge_{q>\alpha_{i}} \mathfrak{c}_{0}{ }^{-1}(g(-, q)) \wedge \bigwedge_{k<i, \alpha_{i}<\alpha_{k}} u_{\alpha_{k}}^{\bullet}\right) \vee \mathfrak{c}_{0}{ }^{-1}(f(p,-))=1,
$$

and that, for each $k<i$ satisfying $\alpha_{k}<\alpha_{i}$,

$$
\left(\bigwedge_{q>\alpha_{i}} \mathfrak{c}_{0}^{-1}(g(-, q)) \wedge \bigwedge_{k<i, \alpha_{i}<\alpha_{k}} u_{\alpha_{k}}^{\bullet}\right) \vee u_{\alpha_{k}}=1
$$

So, it follows from Lemma 4.1 that there exists $u_{\alpha_{i}} \in L_{2}$ such that

$$
\begin{aligned}
\left(q>\alpha_{i}\right) & \Rightarrow\left(\mathfrak{c}_{0}{ }^{-1}(g(-, q)) \vee u_{\alpha_{i}}=1\right), \\
\left(\alpha_{i}<\alpha_{k}\right) & \Rightarrow\left(u_{\alpha_{k}}^{\bullet} \vee u_{\alpha_{i}}=1\right), \text { for all } k<i, \\
\left(p<\alpha_{i}\right) & \Rightarrow\left(\mathfrak{c}_{0}{ }^{-1}(f(p,-)) \vee u_{\alpha_{i}}^{\bullet}=1\right), \\
\left(\alpha_{k}<\alpha_{i}\right) & \Rightarrow\left(u_{\alpha_{k}} \vee u_{\alpha_{i}}^{\bullet}=1\right), \text { for all } k<i
\end{aligned}
$$

This, together with the inductive hypothesis, gives us the required $\left\{u_{\alpha_{k}}\right\}_{k \leq i} \subseteq$ $L_{2}$.

Theorem 4.3. Let $f$ and $g$ be real functions on a biframe $\left(L_{0}, L_{1}, L_{2}\right)$ such that $g$ is $L_{1}$-usc, $f$ is $L_{2}$-lsc and $g \leq f$. Then there exists an $L_{1}$-usc and $L_{2}$-lsc real function $h$ on $L$ satisfying $g \leq h \leq f$, for every such pair $f, g$, if and only if $\left(L_{0}, L_{1}, L_{2}\right)$ is normal.

Proof: $\Rightarrow$ : Let $a \vee b=1$ with $a \in L_{1}$ and $b \in L_{2}$. Then $\chi_{\mathfrak{c}_{0}(a)}$ is $L_{1^{-}}$ usc, $\chi_{\mathfrak{o}_{0}(b)}$ is $L_{2}$-lsc and $\chi_{\mathfrak{c}_{0}(a)} \leq \chi_{\mathfrak{o}_{0}(b)}$. Thus, by hypothesis, there exists an $L_{1}$-usc and $L_{2}$-lsc $h$ such that $\chi_{\mathfrak{c}_{0}(a)} \leq h \leq \chi_{\mathfrak{o}_{0}(b)}$. By Proposition 2.1, $\mathfrak{c}_{0}{ }^{-1} \circ h:\left(\mathfrak{L}(\mathbb{R}), \mathfrak{L}_{l}(\mathbb{R}), \mathfrak{L}_{u}(\mathbb{R})\right) \rightarrow\left(L_{0}, L_{1}, L_{2}\right)$ is a biframe homomorphism. Consider $\left(\mathfrak{c}_{0}{ }^{-1} \circ h\right)\left(-, \frac{1}{2}\right)=v \in L_{1}$ and $\left(\mathfrak{c}_{0}{ }^{-1} \circ h\right)\left(\frac{1}{2},-\right)=u \in L_{2}$. Then $\mathfrak{c}_{0}(u) \wedge \mathfrak{c}_{0}(v)=h(0)=0, \mathfrak{c}_{0}(a) \vee \mathfrak{c}_{0}(u)=\chi_{\mathfrak{c}_{0}(a)}\left(-, \frac{3}{4}\right) \vee h\left(\frac{1}{2},-\right)=1$ and $\mathfrak{c}_{0}(b) \vee \mathfrak{c}_{0}(v)=\chi_{\mathfrak{o}_{0}(b)}\left(\frac{1}{4},-\right) \vee h\left(-, \frac{1}{2}\right)=1$, which means that $u \wedge v=0$ and $a \vee u=1=b \vee v$. This shows the normality of $\left(L_{0}, L_{1}, L_{2}\right)$.

$\Leftarrow$ : Let $g$ be $L_{1}$-usc and let $f$ be $L_{2}$-lsc such that $g \leq f$. By Lemma 4.2 there exists $\left\{u_{\alpha_{k}}\right\}_{k \in \mathbb{N}} \subseteq L_{2}$ satisfying conditions (4.2.1), (4.2.2) and (4.2.3).

Let $q \in \mathbb{Q}$. Then there exists $\alpha_{q} \in \mathbb{Q}$ such that $\alpha_{q}>q$ and it follows that from (4.2.1) that $g(q,-) \leq g(-, q)^{*} \leq \mathfrak{c}_{0}\left(u_{\alpha_{k}}\right)$. Consequently, 
$1=\bigvee_{q \in \mathbb{Q}} g(q,-) \leq \bigvee_{\alpha_{k} \in \mathbb{Q}} \mathfrak{c}_{0}\left(u_{\alpha_{k}}\right)$. Similarly, from (4.2.2) it follows that $1=\bigvee_{q \in \mathbb{Q}} f(-, p) \leq \bigvee_{\alpha_{k} \in \mathbb{Q}} \mathfrak{c}_{0}\left(u_{\alpha_{k}}\right)$. Together with (4.2.3), this means that $\left\{u_{\alpha_{k}}\right\}_{k \in \mathbb{N}}$ is an $L_{2}$-scale thus, by Lemma 3.2, the formulas

$$
h(p,-):=\bigvee_{\alpha_{k}>p} \mathfrak{c}_{0}\left(u_{\alpha_{k}}\right) \quad \text { and } \quad h(-, q):=\bigvee_{\alpha_{k}<q} \mathfrak{c}_{0}\left(u_{\alpha_{k}}^{\bullet}\right)
$$

establish an $L_{1}$-usc and $L_{2}$-lsc real function $h$ on $\left(L_{0}, L_{1}, L_{2}\right)$. Moreover, $g \leq h \leq f:$

(a) Condition (4.2.1) means that $g(-, q) \geq \mathfrak{o}_{0}\left(u_{\alpha_{k}}\right) \geq \mathfrak{c}_{0}\left(u_{\alpha_{k}}^{\bullet}\right)$ for any $q \in \mathbb{Q}$ and each $\alpha_{k}<q$. Consequently,

$$
g(-, q) \geq \bigvee_{\alpha_{k}<q} \mathfrak{c}_{0}\left(u_{\alpha_{k}}^{\bullet}\right)=h(-, q) .
$$

(b) Similarly, by (4.2.2), $f(p,-) \geq \mathfrak{o}_{0}\left(u_{\alpha_{k}}^{\bullet}\right) \geq \mathfrak{c}_{0}\left(u_{\alpha_{k}}\right)$ whenever $p \in \mathbb{Q}$ and $\alpha_{k}>p$. Hence,

$$
f(p,-) \geq \bigvee_{\alpha_{k}>p} \mathfrak{c}_{0}\left(u_{\alpha_{k}}\right)=h(p,-)
$$

\section{Consequences}

5.1. Frames. The particular case $L_{0}=L_{1}=L_{2}$ in Theorem 4.3 gives us immediately the Katětov-Tong-type insertion theorem for frames ([9], [18]).

5.2. Bitopological spaces. Applied to

$$
\left(\mathfrak{T}_{1} \vee \mathfrak{T}_{2}, \mathfrak{T}_{1}, \mathfrak{T}_{2}\right)
$$

for a bitopological space $\left(X, \mathfrak{T}_{1}, \mathfrak{T}_{2}\right)[12]$ one gets the following insertion theorem of Priestley ([21], Theorem 5):

Theorem. Let $f$ and $g$ be real functions on a bitopological space $\left(X, \mathfrak{T}_{1}, \mathfrak{T}_{2}\right)$ such that $g$ is $\mathfrak{T}_{1}$-usc, $f$ is $\mathfrak{T}_{2}$-lsc and $g \leq f$. Then there exists a $\mathfrak{T}_{1}$-usc and $\mathfrak{T}_{2}$-lsc real function $h$ on $X$ satisfying $g \leq h \leq f$, for every such pair $f, g$, if and only if $\left(X, \mathfrak{T}_{1}, \mathfrak{T}_{2}\right)$ is normal.

Proof: We verify the non-trivial implication. Let $\left(X, \mathfrak{T}_{1}, \mathfrak{T}_{2}\right)$ be a normal bitopological space $([12])$ and let $f: X \rightarrow \mathbb{R}$ be a $\mathfrak{T}_{2}$-lsc real function and $g: X \rightarrow \mathbb{R}$ a $\mathfrak{T}_{1}$-usc real function such that $g \leq f$. Note that

$$
\left\{f^{-1}(] p,+\infty[): p \in \mathbb{Q}\right\}
$$


is a lower $\mathfrak{T}_{2}$-scale in the biframe $\left(\mathfrak{T}_{1} \vee \mathfrak{T}_{2}, \mathfrak{T}_{1}, \mathfrak{T}_{2}\right)$ (the only non-trivial fact to be proved is that $\bigvee_{p \in \mathbb{Q}} \mathfrak{a}_{\mathfrak{T}_{1} \vee \mathfrak{T}_{2}}\left(f^{-1}(] p,+\infty[)\right)=1$ which is an immediate consequence of the fact that, for every $p>q, \mathfrak{o}_{\mathfrak{T}_{1} \vee \mathfrak{T}_{2}}\left(f^{-1}(] p,+\infty[)\right) \geq$ $\left.\mathfrak{c}_{\mathfrak{T}_{1} \vee \mathfrak{T}_{2}}\left(f^{-1}(]-\infty, q[)\right)\right)$. Similarly,

$$
\left\{g^{-1}(]-\infty, q[): q \in \mathbb{Q}\right\}
$$

is an upper $\mathfrak{T}_{1}$-scale. Thus, applying Lemma 3.1 , we have a $\mathfrak{T}_{2}$-lsc function $\widetilde{f}: \mathfrak{L}(\mathbb{R}) \rightarrow \mathcal{S}_{0}\left(\mathfrak{T}_{1} \vee \mathfrak{T}_{2}\right)$ given by

$\tilde{f}(p,-)=\bigvee_{r>p} \mathfrak{c}_{\mathfrak{T}_{1} \vee \mathfrak{T}_{2}}\left(f^{-1}(] r,+\infty[)\right) \quad$ and $\quad \tilde{f}(-, q)=\bigvee_{s<q} \mathfrak{o}_{\mathfrak{T}_{1} \vee \mathfrak{T}_{2}}\left(f^{-1}(] s,+\infty[)\right)$ and a $\mathfrak{T}_{1}$-usc function $\widetilde{g}: \mathfrak{L}(\mathbb{R}) \rightarrow \mathcal{S}_{0}\left(\mathfrak{T}_{1} \vee \mathfrak{T}_{2}\right)$ defined by $\widetilde{g}(p,-)=\bigvee_{r>p} \mathfrak{o}_{\mathfrak{T}_{1} \vee \mathfrak{T}_{2}}\left(g^{-1}(]-\infty, r[)\right) \quad$ and $\quad \widetilde{g}(-, q)=\bigvee_{s<q} \mathfrak{c}_{\mathfrak{T}_{1} \vee \mathfrak{T}_{2}}\left(g^{-1}(]-\infty, s[)\right)$. Finally, we have $\widetilde{g} \leq \widetilde{f}$, that is,

$$
\bigvee_{r>p} \mathfrak{o}_{\mathfrak{T}_{1} \vee \mathfrak{T}_{2}}\left(g^{-1}(]-\infty, r[)\right) \leq \bigvee_{r>p} \mathfrak{c}_{\mathfrak{T}_{1} \vee \mathfrak{T}_{2}}\left(f^{-1}(] r,+\infty[)\right)
$$

Indeed: let $r>p$ and take $s$ such that $p<s<r$; then $g^{-1}(]-\infty, r[) \vee$ $g^{-1}(] s,+\infty[)=X$ so $\mathfrak{o}_{\mathfrak{T}_{1} \vee \mathfrak{T}_{2}}\left(g^{-1}(]-\infty, r[)\right) \leq \mathfrak{c}_{\mathfrak{T}_{1} \vee \mathfrak{T}_{2}}\left(g^{-1}(] s,+\infty[)\right)$; finally, since $g \leq f$, then $g^{-1}(] s,+\infty[) \leq f^{-1}(] s,+\infty[)$ and therefore

$$
\mathfrak{c}_{\mathfrak{T}_{1} \vee \mathfrak{T}_{2}}\left(g^{-1}(] s,+\infty[)\right) \leq \mathfrak{c}_{\mathfrak{T}_{1} \vee \mathfrak{T}_{2}}\left(f^{-1}(] s,+\infty[)\right) .
$$

Since $\left(X, \mathfrak{T}_{1}, \mathfrak{T}_{2}\right)$ is normal, the corresponding biframe $\left(\mathfrak{T}_{1} \vee \mathfrak{T}_{2}, \mathfrak{T}_{1}, \mathfrak{T}_{2}\right)$ is normal and, by Theorem 4.3, there exists an $L_{1}$-usc and $L_{2}$-lsc $\widetilde{h}$ such that $\widetilde{g} \leq \widetilde{h} \leq \widetilde{f}$. By Proposition 2.1, $\widetilde{h}: \mathfrak{L}(\mathbb{R}) \rightarrow \mathcal{S}_{0}\left(\mathfrak{T}_{1} \vee \mathfrak{T}_{2}\right)$ is continuous in $\mathfrak{T}_{1} \vee \mathfrak{T}_{2}$ and

$$
\left(\mathfrak{c}_{\mathfrak{T}_{1} \vee \mathfrak{T}_{2}}\right)^{-1} \circ \widetilde{h}:\left(\mathfrak{L}(\mathbb{R}), \mathfrak{L}_{l}(\mathbb{R}), \mathfrak{L}_{u}(\mathbb{R})\right) \rightarrow\left(\mathfrak{T}_{1} \vee \mathfrak{T}_{2}, \mathfrak{T}_{1}, \mathfrak{T}_{2}\right)
$$

is a biframe homomorphism. By Proposition 4 of [1] there is a continuous function $h:\left(X, \mathfrak{T}_{1} \vee \mathfrak{T}_{2}\right) \rightarrow \mathbb{R}$ satisfying, for each $p, q \in \mathbb{Q}$,

$$
p<h(x)<q \quad \Leftrightarrow \quad x \in\left(\left(\mathfrak{c}_{\mathfrak{T}_{1} \vee \mathfrak{T}_{2}}\right)^{-1} \circ \widetilde{h}\right)(p, q) .
$$

We have immediately that $g \leq h \leq f, h$ is $\mathfrak{T}_{1}$-usc and $\mathfrak{T}_{2}$-lsc (since $h^{-1}(]-\infty, q[) \subseteq\left(\left(\mathfrak{c}_{\mathfrak{T}_{1} \vee \mathfrak{T}_{2}}\right)^{-1} \circ \widetilde{h}\right)(-, q) \in \mathfrak{T}_{1}$ and $h^{-1}(] p,+\infty[) \subseteq\left(\left(\mathfrak{c}_{\mathfrak{T}_{1} \vee \mathfrak{T}_{2}}\right)^{-1} \circ\right.$ $\left.\widetilde{h})(p,-) \in \mathfrak{T}_{2}\right)$. 
Remark. Similarly, the monotone version of Theorem 4.3 (following and extending the lines of [5]) would give us Theorem 1 of [16].

5.3. Ordered topological spaces. An ordered topological space $(X, \mathfrak{T}, \leq)$ is:

(1) normal (normally ordered in $[17,21]$ ) if, given disjoint closed subsets $F_{1}, F_{2}$ of $X$ with $F_{1}$ decreasing and $F_{2}$ increasing, there exist disjoint open sets $G_{1}, G_{2}$ such that $G_{1}$ is decreasing, $G_{2}$ increasing and $F_{j} \subseteq$ $G_{j}, j=1,2$

(2) a $N_{i}$-space [21] if, given disjoint closed subsets $F_{1}, F_{2}$ of $X$ with $F_{2}$ increasing, there exist disjoint open sets $G_{1}, G_{2}$ such that $G_{1}$ is decreasing, $G_{2}$ is increasing and $F_{j} \subseteq G_{j}, j=1,2$;

(3) a $N_{d}$-space [21] if, given disjoint closed subsets $F_{1}, F_{2}$ of $X$ with $F_{1}$ decreasing, there exist disjoint open sets $G_{1}, G_{2}$ such that $G_{1}$ is decreasing, $G_{2}$ is increasing and $F_{j} \subseteq G_{j}, j=1,2$;

(4) a $N$-space [21] if, given closed sets $F_{1}, F_{2}$ satisfying $a_{1} \ngtr a_{2}$ for every $a_{1} \in F_{1}$ and $a_{2} \in F_{2}$, there exist disjoint open sets $G_{1}, G_{2}$ such that $G_{1}$ is decreasing, $G_{2}$ is increasing and $F_{j} \subseteq G_{j}, j=1,2$.

The sets $\uparrow \mathfrak{T}$ (of open increasing sets) and $\downarrow \mathfrak{T}$ (of open decreasing sets) define two topologies on $X$ and therefore the triples $(X, \uparrow \mathfrak{T}, \downarrow \mathfrak{T})$ and $(X, \downarrow \mathfrak{T}, \uparrow \mathfrak{T})$ are bitopological spaces. The following is obvious:

- $(X, \mathfrak{T}, \leq)$ is normal iff $(X, \uparrow \mathfrak{T}, \downarrow \mathfrak{T})$ is normal iff $(X, \downarrow \mathfrak{T}, \uparrow \mathfrak{T})$ is normal.

- If $(X, \mathfrak{T}, \leq)$ is $N_{i}$ then $(X, \mathfrak{T}, \downarrow \mathfrak{T})$ and $(X, \downarrow \mathfrak{T}, \mathfrak{T})$ are normal.

- If $(X, \mathfrak{T}, \leq)$ is $N_{d}$ then $(X, \uparrow \mathfrak{T}, \mathfrak{T})$ and $(X, \mathfrak{T}, \uparrow \mathfrak{T})$ are normal.

- $f:(X, \downarrow \mathfrak{T}) \rightarrow \mathbb{R}$ is usc iff $f:(X, \mathfrak{T}) \rightarrow \mathbb{R}$ is usc and $f$ is monotone.

- $f:(X, \uparrow \mathfrak{T}) \rightarrow \mathbb{R}$ is lsc iff $f:(X, \mathfrak{T}) \rightarrow \mathbb{R}$ is lsc and $f$ is monotone.

Furthermore:

(*) An ordered topological space $(X, \mathfrak{T}, \leq)$ is an $N$-space iff it is simultaneously an $N_{i}$-space and an $N_{d}$-space and $\leq$ is a closed partial order [21, Lemma 3].

Using these results one can immediately obtain the following theorems as particular cases of our Theorem 4.3 above:

Theorem 1. Let $f$ and $g$ be monotone real functions on an ordered topological space $(X, \mathfrak{T}, \leq)$ such that $g$ is usc, $f$ is lsc and $g \leq f$. Then there exists a continuous monotone real function $h$ on $X$ satisfying $g \leq h \leq f$, for every such pair $f$ and $g$, if and only if $(X, \mathfrak{T}, \leq)$ is normal. 
Proof: Use the properties above and apply Theorem 4.3 to the biframe $(\downarrow \mathfrak{T} \vee$ $\uparrow \mathfrak{T}, \downarrow \mathfrak{T}, \uparrow \mathfrak{T})$.

Theorem 2. Let $f$ and $g$ be real functions on an ordered topological space $(X, \mathfrak{T}, \leq)$ such that $g$ is usc, $f$ is lsc, $g \leq f$ and $f$ is monotone. Then there exists a continuous monotone real function $h$ on $X$ satisfying $g \leq h \leq f$, for every such pair $f$ and $g$, if and only if $(X, \mathfrak{T}, \leq)$ is $N_{i}$.

Proof: Use the properties above and apply Theorem 4.3 to the biframe $(\mathfrak{T} \vee \uparrow \mathfrak{T}, \mathfrak{T}, \uparrow \mathfrak{T})$

Theorem 3. Let $f$ and $g$ be real functions on an ordered topological space $(X, \mathfrak{T}, \leq)$ such that $g$ is usc, $f$ is $l s c, g \leq f$ and $g$ is monotone. Then there exists a continuous monotone real function $h$ on $X$ satisfying $g \leq h \leq f$, for every such pair $f$ and $g$, if and only if $(X, \mathfrak{T}, \leq)$ is $N_{d}$.

Proof: Use the properties above and apply Theorem 4.3 to the biframe $(\downarrow \mathfrak{T} \vee \mathfrak{T}, \downarrow \mathfrak{T}, \mathfrak{T})$

Then, immediately, using property $(*)$, Theorems 2 and 3 produce:

Corollary 4. Let $f$ and $g$ be monotone real functions on an ordered topological space $(X, \mathfrak{T}, \leq)$ such that $g$ is usc, $f$ is lsc and $g \leq f$. If $(X, \mathfrak{T}, \leq)$ is an $N$-space then there exists a continuous monotone real function $h$ on $X$ satisfying $g \leq h \leq f$. The converse is true if $\leq$ is closed.

Remark. Theorems 1 and 2 are, respectively, Theorems 1 and 2 of Priestley [21]. Note that, as in the bitopological case (subsection 5.2 above), the former provides as a corollary Nachbin's extension to normal ordered topological spaces of Urysohn's Lemma, with $g, f$ characteristic functions of appropriate sets $([17]$, p. 30).

5.4. Urysohn-type lemma. Let $\left(L_{0}, L_{1}, L_{2}\right)$ be a normal biframe, $a \vee$ $b=1$ with $a \in L_{1}$ and $b \in L_{2}$. Then $\chi_{\mathfrak{c}_{0}(a)}$ is $L_{1}$-usc, $\chi_{\mathfrak{o}_{0}(b)}$ is $L_{2}$-lsc and $\chi_{\mathfrak{c}_{0}(a)} \leq \chi_{\mathfrak{o}_{0}(b)}$. Therefore there exists an $L_{1}$-usc and $L_{2}$-lsc function $h$ such that $\chi_{\mathfrak{c}_{0}(a)} \leq h \leq \chi_{\mathfrak{o}_{0}(b)}$. By Proposition 2.1,

$$
\mathfrak{c}_{0}{ }^{-1} \circ h:\left(\mathfrak{L}(\mathbb{R}), \mathfrak{L}_{l}(\mathbb{R}), \mathfrak{L}_{u}(\mathbb{R})\right) \rightarrow\left(L_{0}, L_{1}, L_{2}\right)
$$

is a biframe homomorphism. Further, $\chi_{\mathfrak{c}_{0}(a)} \leq h$ means that $\chi_{\mathfrak{c}_{0}(a)}(-, q) \leq$ $h(-, q)$ for every $0<q \leq 1$, that is, $h(-, 1) \leq \mathfrak{c}_{0}(a)$. Similarly, $h \leq \chi_{\mathfrak{o}_{0}(b)}$ implies $h(0,-) \leq \mathfrak{c}_{0}(b)$. Hence $\left(\mathfrak{c}_{0}{ }^{-1} \circ h\right)(-, 1) \leq a$ and $\left(\mathfrak{c}_{0}{ }^{-1} \circ h\right)(0,-) \leq b$. We 
have just obtained the Urysohn's Lemma for normal biframes of Schauerte [23, Proposition 6.2]:

Proposition. A biframe $\left(L_{0}, L_{1}, L_{2}\right)$ is normal if and only if, whenever $a \vee b=1$ for $a \in L_{1}$ and $b \in L_{2}$, there exists a biframe homomorphism $h:\left(\mathfrak{L}(\mathbb{R}), \mathfrak{L}_{l}(\mathbb{R}), \mathfrak{L}_{u}(\mathbb{R})\right) \rightarrow\left(L_{0}, L_{1}, L_{2}\right)$ such that $h(-, 1) \leq a$ and $h(0,-) \leq$ $b$.

5.5. Tietze-type extension theorem. Given a sub-bilocale $\left(S_{0}, S_{1}, S_{2}\right)$ of $\left(L_{0}, L_{1}, L_{2}\right)$, we say that an $S_{1}$-usc and $S_{2}$-lsc real function $f$ on $\left(S_{0}, S_{1}, S_{2}\right)$ has a continuous extension to $\left(L_{0}, L_{1}, L_{2}\right)$ if there exists an $L_{1}$-usc and $L_{2^{-}}$ lsc real function $\tilde{f}$ on $\left(L_{0}, L_{1}, L_{2}\right)$ such that $\tilde{f}(p, q) \vee S_{0}=f(p, q)$ for every $p, q \in \mathbb{Q}$. This means precisely that the diagram

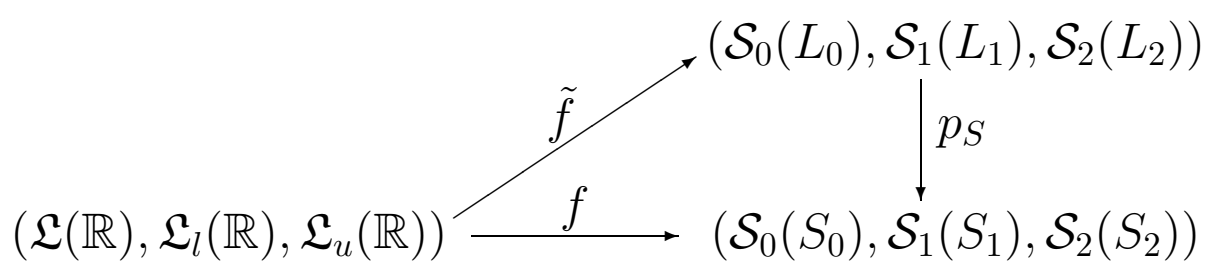

(with $p_{S}$ defined by $p_{S}(T)=T \vee S_{0}$ ) is commutative.

Then we get what appears to be a new result for biframes (the Tietze-type extension theorem for normal biframes) that generalizes at once the results for frames [8], bitopological spaces [12] and ordered topological spaces [17, 21] (the details of the proof are rather technical and will appear elsewhere):

Theorem. A biframe $\left(L_{0}, L_{1}, L_{2}\right)$ is normal if and only if for every closed sub-bilocale $\left(S_{0}, S_{1}, S_{2}\right)$, each $S_{1}$-usc and $S_{2}$-lsc real function on $\left(S_{0}, S_{1}, S_{2}\right)$ has a continuous extension to $\left(L_{0}, L_{1}, L_{2}\right)$.

\section{Stone-type insertion}

We say that a biframe $\left(L_{0}, L_{1}, L_{2}\right)$ is extremally disconnected (cf. [13] for the corresponding bitopological notion) if whenever $a \wedge b=0$ for some $a \in L_{1}$ and $b \in L_{2}$ there exist $u \in L_{2}$ and $v \in L_{1}$ such that $u \vee v=1$ and $a \wedge u=0=b \wedge v$.

Remark 6.1. Note that a biframe is extremally disconnected if and only if $a^{\bullet \bullet} \vee a^{\bullet}=1$ for every $a \in L_{1}$ (or, equivalently, if $b^{\bullet} \vee b^{\bullet \bullet}=1$ for every $b \in L_{2}$ ). Indeed: 
If $\left(L_{0}, L_{1}, L_{2}\right)$ is extremally disconnected then, since $a^{\bullet \bullet} \wedge a^{\bullet}=0$, there exist $u \in L_{2}$ and $v \in L_{1}$ such that $u \vee v=1$ and $a^{\bullet \bullet} \wedge u=0=a^{\bullet} \wedge v$. Evidently, $1=u \vee v \leq a^{\bullet} \vee a^{\bullet \bullet}$. Conversely, let $a \wedge b=0$ with $a \in L_{1}$ and $b \in L_{2}$. Then $b \leq a^{\bullet}$. Take $u=a^{\bullet} \in L_{2}$ and $v=a^{\bullet \bullet} \in L_{1}$. Clearly, $u \vee v=1$, $a \wedge u=0$ and $b \wedge v=b \wedge a^{\bullet \bullet} \leq a^{\bullet} \wedge a^{\bullet \bullet}=0$.

Given a biframe $\left(L_{0}, L_{1}, L_{2}\right)$ and $S \in \mathcal{S}_{0}\left(L_{0}\right)$, we denote by $\operatorname{cl}_{i}(S)(i=1,2)$ the closure of $S$ in $\mathcal{S}_{i}\left(L_{i}\right)$, that is,

$$
\operatorname{cl}_{i}(S)=\bigvee\left\{\mathfrak{c}_{0}(a) \mid a \in L_{i}, \mathfrak{c}_{0}(a) \leq S\right\} .
$$

Note that $\operatorname{cl}_{i}\left(\mathfrak{o}_{0}(a)\right)=\mathfrak{c}_{0}\left(a^{\bullet}\right)$ for each $a \in L_{i}(i=1,2)$.

We need now to extend the lower and upper regularizations $[6,8]$ of frame real functions to biframe real functions. Let

$$
f:\left(\mathfrak{L}(\mathbb{R}), \mathfrak{L}_{l}(\mathbb{R}), \mathfrak{L}_{u}(\mathbb{R})\right) \rightarrow\left(\mathcal{S}_{0}\left(L_{0}\right), \mathcal{S}_{1}\left(L_{1}\right), \mathcal{S}_{2}\left(L_{2}\right)\right) .
$$

The lower regularization $f^{\circ}$ of $f$ is defined by:

$$
f^{\circ}(p,-)=\bigvee_{r>p} \operatorname{cl}_{2}(f(r,-)) \text { and } f^{\circ}(-, q)=\bigvee_{s<q}\left(\operatorname{cl}_{2}(f(s,-))\right)^{*} .
$$

Dually, the upper regularization $f^{-}$of $f$ is defined by

$$
f^{-}(p,-)=\bigvee_{r>p}\left(\operatorname{cl}_{1}(f(-, r))\right)^{*} \quad \text { and } \quad f^{-}(-, q)=\bigvee_{s<q} \operatorname{cl}_{1}(f(-, s)) .
$$

Properties 6.2. The following hold for all real functions $f, g$ in $\left(L_{0}, L_{1}, L_{2}\right)$ :

(1) $f^{\circ} \leq f$

(2) $f^{\circ}$ is $L_{2}$-lsc.

(3) If $g$ is $L_{2}$-lsc and $g \leq f$ then $g \leq f^{\circ}$.

(4) $f \leq f^{-}$.

(5) $f^{-}$is $L_{1}$-usc.

(6) If $g$ is $L_{1}$-usc and $f \leq g$ then $f^{-} \leq g$.

Proof: (1) For each $p \in \mathbb{Q}, f^{\circ}(p,-)=\bigvee_{r>p} \mathrm{cl}_{2}(f(r,-)) \leq \bigvee_{r>p} f(r,-)=$ $f(p,-)$.

(2) It is obvious.

(3) The fact that $g$ is $L_{2}$-lsc means that $g(p,-)=\mathfrak{c}_{0}\left(b_{p}\right)$ for some $b_{p} \in L_{2}$. Then $\mathfrak{c}_{0}\left(b_{p}\right)=g(p,-) \leq f(p,-)$, which implies that $g(p,-) \leq \operatorname{cl}_{2}(f(p,-))$. Therefore

$$
f^{\circ}(p,-)=\bigvee_{r>p} \operatorname{cl}_{2}(f(r,-)) \geq \bigvee_{r>p} g(r,-)=g(p,-)
$$


Properties (4)-(6) can be proved similarly.

Lemma 6.3. Let $g$ be an $L_{1}$-usc real function on $\left(L_{0}, L_{1}, L_{2}\right)$ and let $g(-, q)=$ $\mathfrak{c}_{0}\left(a_{q}\right)$ with $a_{q} \in L_{1}$. Then:

(1) $g^{\circ}(p,-) \geq \bigvee_{r>p} \mathfrak{c}_{0}\left(a_{r}^{\bullet}\right)$.

(2) $g^{\circ-}(p,-) \leq \bigvee_{r>p} \mathfrak{o}_{0}\left(\bigvee_{s<r} a_{s}^{\bullet \bullet}\right)$

(3) If $a_{q}^{\bullet} \vee a_{q}^{\bullet \bullet}=1$ for every $q \in \mathbb{Q}$, then $g^{\circ-}=g^{\circ}$.

Proof: (1) We need to check that $\bigvee_{r>p} \mathfrak{c}_{0}\left(a_{r}^{\bullet}\right) \leq \bigvee_{r>p} \operatorname{cl}_{2}(g(r,-))$. For each $r>p$ consider $t$ such that $p<t<r$. Then $g(t,-) \vee \mathfrak{c}_{0}\left(a_{r}\right)=1$, which implies $g(t,-) \geq \mathfrak{o}_{0}\left(a_{r}\right) \geq \operatorname{cl}_{2}\left(\mathfrak{o}_{0}\left(a_{r}\right)\right)=\mathfrak{c}_{0}\left(a_{r}^{\bullet}\right)$. Hence $\mathfrak{c}_{0}\left(a_{r}^{\bullet}\right) \leq g(t,-)$ and, consequently, $\mathfrak{c}_{0}\left(a_{r}^{\bullet}\right) \leq \operatorname{cl}_{2}(g(t,-))$.

(2) Since $g^{\circ-}(p,-)=\bigvee_{r>p}\left(\operatorname{cl}_{1}\left(g^{\circ}(-, r)\right)\right)^{*}$, we have to show that

$$
\bigvee_{r>p}\left(\operatorname{cl}_{1}\left(g^{\circ}(-, r)\right)\right)^{*} \leq \bigvee_{r>p} \mathfrak{o}_{0}\left(\bigvee_{s<r} a_{s}^{\bullet \bullet}\right)
$$

For that it suffices to check that, for any $r>p, \mathfrak{c}_{0}\left(\bigvee_{s<r} a_{s}^{\bullet \bullet}\right) \leq \operatorname{cl}_{1}\left(g^{\circ}(-, r)\right)$. Since $g(s,-) \leq g(-, s)^{*}=\mathfrak{o}_{0}\left(a_{s}\right)$, we have $\operatorname{cl}_{2}(g(s,-)) \leq \operatorname{cl}_{2}\left(\mathfrak{o}_{0}\left(a_{s}\right)\right)=\mathfrak{c}_{0}\left(a_{s}^{\bullet}\right)$, from which it follows that $\mathfrak{c}_{0}\left(a_{s}^{\bullet \bullet}\right)=\operatorname{cl}_{1}\left(\mathfrak{o}\left(a_{s}^{\bullet}\right)\right) \leq \mathfrak{o}\left(a_{s}^{\bullet}\right) \leq \operatorname{cl}_{2}(g(s,-))^{*}$. Hence

$$
\mathfrak{c}_{0}\left(\bigvee_{s<r} a_{s}^{\bullet \bullet}\right)=\bigvee_{s<r} \mathfrak{c}_{0}\left(a_{s}^{\bullet \bullet}\right) \leq \bigvee_{s<r} \operatorname{cl}_{2}(g(s,-))^{*}=g^{\circ}(-, r)
$$

which ensures that $\mathfrak{c}_{0}\left(\bigvee_{s<r} a_{s}^{\bullet \bullet}\right) \leq \operatorname{cl}_{1}\left(g^{\circ}(-, r)\right)$.

(3) By Properties 6.2 (4), it suffices to check that $g^{\circ-} \leq g^{\circ}$. Using (1) and (2), we only need to check that

$$
\bigvee_{r>p} \mathfrak{o}_{0}\left(\bigvee_{s<r} a_{s}^{\bullet \bullet \bullet}\right) \leq \bigvee_{r>p} \mathfrak{c}_{0}\left(a_{r}^{\bullet}\right)
$$

So, let $r>p$ and take $t$ such that $p<t<r$. Since $a_{t}^{\bullet \bullet} \vee a_{t}^{\bullet}=1$ we have $\mathfrak{o}_{0}\left(a_{t}^{\bullet \bullet \bullet}\right) \leq \mathfrak{c}_{0}\left(a_{t}^{\bullet}\right)$. This means that $\mathfrak{o}_{0}\left(\bigvee_{s<r} a_{s}^{\bullet \bullet}\right) \leq \mathfrak{o}_{0}\left(a_{t}^{\bullet \bullet}\right) \leq \mathfrak{c}_{0}\left(a_{t}^{\bullet}\right)$ and the required inequality follows.

Theorem 6.4. Let $f$ and $g$ be real functions on a biframe $\left(L_{0}, L_{1}, L_{2}\right)$ such that $g$ is $L_{1}$-usc, $f$ is $L_{2}$-lsc and $f \leq g$. Then there exists an $L_{1}$-usc and $L_{2}$-lsc real function $h$ on $\left(L_{0}, L_{1}, L_{2}\right)$ satisfying $f \leq h \leq g$, for every such pair $f, g$, if and only if $L$ is extremally disconnected.

Proof: $\Rightarrow$ : Let $a \wedge b=0$ with $a \in L_{1}$ and $b \in L_{2}$. Then $\chi_{\mathfrak{c}_{0}(a)}$ is $L_{1}$-usc, $\chi_{\mathfrak{o}_{0}(b)}$ is $L_{2}$-lsc and $\chi_{\mathfrak{o}_{0}(b)} \leq \chi_{\mathfrak{c}_{0}(a)}$. Thus, by hypothesis, there exists an $L_{1}$-usc and 
$L_{2}$-lsc $h$ such that $\chi_{\mathfrak{o}_{0}(b)} \leq h \leq \chi_{\mathfrak{c}_{0}(a)}$. By Proposition 2.1,

$$
\mathfrak{c}_{0}{ }^{-1} \circ h:\left(\mathfrak{L}(\mathbb{R}), \mathfrak{L}_{l}(\mathbb{R}), \mathfrak{L}_{u}(\mathbb{R})\right) \rightarrow\left(L_{0}, L_{1}, L_{2}\right)
$$

is a biframe homomorphism. Consider $\left(\mathfrak{c}_{0}{ }^{-1} \circ h\right)\left(-, \frac{1}{2}\right)=v \in L_{1}$ and $\left(\mathfrak{c}_{0}{ }^{-1} \circ\right.$ $h)\left(\frac{1}{2},-\right)=u \in L_{2}$. Then $\mathfrak{c}_{0}(u) \vee \mathfrak{c}_{0}(v)=h(1)=1, \mathfrak{c}_{0}(a) \wedge \mathfrak{c}_{0}(u)=\chi_{\mathfrak{c}_{0}(a)}\left(-, \frac{1}{2}\right) \wedge$ $h\left(\frac{1}{2},-\right) \leq \chi_{\mathfrak{c}_{0}(a)}\left(-, \frac{1}{2}\right) \wedge \chi_{\mathfrak{c}_{0}(a)}\left(\frac{1}{2},-\right)=0$ and $\mathfrak{c}_{0}(b) \wedge \mathfrak{c}_{0}(v)=\chi_{\mathfrak{o}_{0}(b)}\left(\frac{1}{2},-\right) \wedge$ $h\left(-, \frac{1}{2}\right) \leq h\left(\frac{1}{2},-\right) \wedge h\left(-, \frac{1}{2}\right)=0$, which means that $u \vee v=1$ and $a \wedge u=$ $0=b \wedge v$. This shows the extremal disconnectedness of $\left(L_{0}, L_{1}, L_{2}\right)$.

$\Leftarrow$ : Let $f$ and $g$ be real functions on a biframe $\left(L_{0}, L_{1}, L_{2}\right)$ such that $g$ is $L_{1^{-}}$ usc, $f$ is $L_{2}$-lsc and $f \leq g$. By Properties $6.2, f \leq g^{\circ} \leq g$. Since $g$ is $L_{1}$-usc, we have $g(-, q)=\mathfrak{c}_{0}\left(a_{q}\right)$ for some $a_{q} \in L_{1}$. The extremal disconnectedness of $\left(L_{0}, L_{1}, L_{2}\right)$ implies, by Lemma 6.3(3), that $g^{\circ}=g^{\circ-}$. We have here the required $h$ (since $g^{\circ}$ is $L_{2}$-lsc and $g^{\circ-}$ is $L_{1}$-usc).

\section{Consequences}

7.1. Frames. The particular case $L_{0}=L_{1}=L_{2}$ in Theorem 6.4 gives us immediately the Stone-type insertion theorem for frames $[14,6]$.

7.2. Bitopological spaces. Applied to

$$
\left(\mathfrak{T}_{1} \vee \mathfrak{T}_{2}, \mathfrak{T}_{1}, \mathfrak{T}_{2}\right)
$$

for a bitopological space $\left(X, \mathfrak{T}_{1}, \mathfrak{T}_{2}\right)$ one gets the following insertion theorem for bitopological spaces that appears to be new:

Theorem. Let $f$ and $g$ be real functions on a bitopological space $\left(X, \mathfrak{T}_{1}, \mathfrak{T}_{2}\right)$ such that $g$ is $\mathfrak{T}_{1}$-usc, $f$ is $\mathfrak{T}_{2}$-lsc and $f \leq g$. Then there exists a $\mathfrak{T}_{1}$-usc and $\mathfrak{T}_{2}$-lsc real function $h$ on $X$ satisfying $f \leq h \leq g$, for every such pair $f, g$, if and only if $\left(X, \mathfrak{T}_{1}, \mathfrak{T}_{2}\right)$ is extremally disconnected.

Proof: Similar to the proof of Theorem 5.2.

This provides immediately as a corollary (with $f, g$ characteristic functions of appropriate sets) the Urysohn-type lemma for extremally disconnected bitopological spaces of Sarma [22, Theorem 2.4]. 
7.3. Ordered topological spaces. Given an ordered topological space $(X, \mathfrak{T}, \leq)$ and a subset $E$ of $X$, let $\mathrm{cl} \uparrow(E)$ denote the smallest closed increasing subset of $X$ containing $E$ and let $\operatorname{cl} \downarrow(E)$ denote the smallest closed decreasing subset of $X$ containing $E$. The ordered space $(X, \mathfrak{T}, \leq)$ is extremally disconnected (extremally order-disconnected in [3]) if for each increasing open subset $G_{1}$ of $X$ the set $\operatorname{cl} \uparrow\left(G_{1}\right)$ is open and for each decreasing open subset $G_{2}$ of $X$ the set $\operatorname{cl} \downarrow\left(G_{2}\right)$ is open. After realizing that

$(X, \mathfrak{T}, \leq)$ is extremally disconnected iff $(X, \uparrow \mathfrak{T}, \downarrow \mathfrak{T})$ is extremally disconnected iff $(X, \downarrow \mathfrak{T}, \uparrow \mathfrak{T})$ is extremally disconnected,

one can obtain immediately the following theorem as a particular case of our Theorem 6.4 above (cf. [3]):

Theorem. Let $f$ and $g$ be monotone real functions on a ordered topological space $(X, \mathfrak{T}, \leq)$ such that $g$ is usc, $f$ is lsc and $f \leq g$. Then there exists a continuous monotone function $h$ on $X$ satisfying $f \leq h \leq h$, for every such pair $f, g$, if and only if $(X, \mathfrak{T}, \leq)$ is extremally disconnected.

7.4. Urysohn-type lemma. Let $\left(L_{0}, L_{1}, L_{2}\right)$ be a normal biframe, $a \wedge$ $b=0$ with $a \in L_{1}$ and $b \in L_{2}$. Then $\chi_{\mathfrak{c}_{0}(a)}$ is $L_{1}$-usc, $\chi_{\mathfrak{o}_{0}(b)}$ is $L_{2}$-lsc and $\chi_{\mathfrak{o}_{0}(b)} \leq \chi_{\mathfrak{c}_{0}(a)}$. Therefore there exists an $L_{1}$-usc and $L_{2}$-lsc function $h$ such that $\chi_{\mathfrak{o}_{0}(b)} \leq h \leq \chi_{\mathfrak{c}_{0}(a)}$. By Proposition 2.1,

$$
\mathfrak{c}_{0}{ }^{-1} \circ h:\left(\mathfrak{L}(\mathbb{R}), \mathfrak{L}_{l}(\mathbb{R}), \mathfrak{L}_{u}(\mathbb{R})\right) \rightarrow\left(L_{0}, L_{1}, L_{2}\right)
$$

is a biframe homomorphism. Further, $\chi_{\mathfrak{o}_{0}(b)} \leq h$ means that $\chi_{\mathfrak{o}_{0}(b)}(-, q) \leq$ $h(-, q)$ for every $0<q \leq 1$, that is, $\mathfrak{o}_{0}(b) \leq h(-, 1)$. Similarly, $h \leq \chi_{\mathfrak{c}_{0}(a)}$ implies $h(0,-) \leq \mathfrak{o}_{0}(a)$. Since $\left(\mathfrak{c}_{0}^{-1} \circ h\right)(-, 1)=u$ for some $u \in L_{1}$ and $\left(\mathfrak{c}_{0}{ }^{-1} \circ h\right)(0,-)=v$ for some $v \in L_{2}$, we have $\mathfrak{o}_{0}(b) \leq \mathfrak{c}_{0}(u)$ and $\mathfrak{c}_{0}(v) \leq \mathfrak{o}_{0}(a)$, that is, $b \vee u=1$ and $a \wedge v=0$. We have just obtained the following separation-type lemma for extremally disconnected biframes, which appears to be new:

Proposition. A biframe $\left(L_{0}, L_{1}, L_{2}\right)$ is extremally disconnected if and only if whenever $a \wedge b=0$ for $a \in L_{1}$ and $b \in L_{2}$, there exists a biframe homomorphism $h:\left(\mathfrak{L}(\mathbb{R}), \mathfrak{L}_{l}(\mathbb{R}), \mathfrak{L}_{u}(\mathbb{R})\right) \rightarrow\left(L_{0}, L_{1}, L_{2}\right)$ such that $a \wedge h(0,-)=0$ and $b \vee h(-, 1)=1$.

7.5. Tietze-type extension theorem. Similarly to 5.5, we can also produce an extension-type result for extremally disconnected biframes: 
Theorem. A biframe $\left(L_{0}, L_{1}, L_{2}\right)$ is extremally disconnected if and only if for every open sub-bilocale $\left(S_{0}, S_{1}, S_{2}\right)$, each $S_{1}$-usc and $S_{2}$-lsc real function on $\left(S_{0}, S_{1}, S_{2}\right)$ has a continuous extension to $\left(L_{0}, L_{1}, L_{2}\right)$.

This is new for biframes and extends at once the results for frames [14, 6], bitopological spaces [22] and ordered topological spaces.

\section{References}

[1] B. Banaschewski, The Real Numbers in Pointfree Topology, Textos de Matemática, Vol. 12 (University of Coimbra, 1997).

[2] B. Banaschewski, G.C.L. Brümmer, K.A. Hardie, Biframes and bispaces, Quaestiones Math. 6 (1983) 13-25.

[3] D.A. Edwards, A separation theorem for semicontinuous functions on preordered topological spaces, Arch. Math. 84 (2005) 559-567.

[4] M.J. Ferreira, J. Gutiérrez García, J. Picado, Completely normal frames and real-valued functions, to appear in Topology Appl.

[5] J. Gutiérrez García, T. Kubiak, J. Picado, Monotone insertion and monotone extension of frame homomorphisms, J. Pure Appl. Algebra 212 (2008) 955-968.

[6] J. Gutiérrez García, T. Kubiak, J. Picado, Lower and upper regularizations of frame semicontinuous real functions, to appear in Algebra Universalis (2009), doi:10.1007/500012-0092102-8.

[7] J. Gutiérrez García, T. Kubia, J. Picado, Pointfree forms of Dowker's and Michael's insertion theorems, J. Pure Appl. Algebra 213 (2009) 98-108.

[8] J. Gutiérrez García, T. Kubiak, J. Picado, Localic real functions: a general setting, to appear in J. Pure Appl. Algebra (2009), doi:10.1016/j.jpaa.2008.11.004.

[9] J. Gutiérrez García, J. Picado, On the algebraic representation of semicontinuity, J. Pure Appl. Algebra 210 (2007) 299-306.

[10] P.T. Johnstone, Stone Spaces, Cambridge Univ. Press, Cambridge, 1982.

[11] M. Katětov, On real-valued functions in topological spaces, Fund. Math. 38 (1951) 85-91; Correction, Fund. Math. 40 (1953) 139-142.

[12] J.C. Kelly, Bitopological spaces, Proc. London Math. Soc. (3) 13 (1963) 71-89.

[13] S. Lal, Pairwise concepts in bitopological spaces, J. Austral. Math. Soc. (Ser.A) 26 (1978) 241-250.

[14] Y.-M. Li, Z.-H. Li, Constructive insertion theorems and extension theorems on extremally disconnected frames, Algebra Univers. 44 (2000) 271-281.

[15] Y.-M. Li, G.-J. Wang, Localic Katětov-Tong insertion theorem and localic Tietze extension theorem, Comment. Math. Univ. Carolinae 38 (1997) 801-814.

[16] J. Marín, S. Romaguera, Pairwise monotonically normal spaces, Comment. Math. Univ. Carolinae 32 (1991) 567-579.

[17] L. Nachbin, Topology and Order, Van Nostrand Mathematical Studies 4, Princeton, 1965.

[18] J. Picado, A new look at localic interpolation theorems, Topology Appl. 153 (2006) 3203-3218.

[19] J. Picado, A. Pultr, Locales Treated Mostly in a Covariant Way, Textos de Matemática, Vol. 41 (University of Coimbra, 2008).

[20] J. Picado, A. Pultr, A. Tozzi, Locales, in: M.C. Pedicchio, W. Tholen (Eds.), Categorical Foundation - Special Topics in Order, Algebra and Sheaf Theory, Encyclopedia of Mathematics and its Applications, Vol. 97, Cambridge Univ. Press, Cambridge, 2004, pp. 49-101. 
[21] H. Priestley, Separation theorems for semi-continuous functions on normally ordered topological spaces, J. London Math. Soc. (2) 3 (1971) 371-377.

[22] R.D. Sarma, A note on extremal disconnectedness in bitopological spaces, Acta Math. Hungar. 120 (2008) 141-146.

[23] A. Schauerte, Biframes, Ph.D. Thesis, McMaster University, 1992.

[24] A. Schauerte, Normality for biframes, Applied Categ. Structures 3 (1995) 1-9.

[25] H. Tong, Some characterizations of normal and perfectly normal spaces, Duke J. Math. 19 (1952) 289-292.

[26] M.H. Stone, Boundedness properties in function-lattices, Canad. J. Math. 1 (1949) 176-186.

MARIA Jỗo Ferreira

CMUC, Department of Mathematics, University of Coimbra, 3001-454 Coimbra, Portugal

E-mail address: mjrf@mat.uc.pt

Javier Gutiérrez García

Departamento de Matemáticas, Universidad del País Vasco-Euskal Herriko Unibertsitatea, Apartado 644, 48080, Bilbao, Spain

E-mail address: javier.gutierrezgarcia@lg.ehu.es

JoRGE PICADO

CMUC, Department of Mathematics, University of Coimbra, 3001-454 Coimbra, Portugal

E-mail address: picado@mat.uc.pt 